\title{
PPreDeCon: A Parallel version of Preference Density Connected Clustering Algorithm
}

\author{
Raheleh Biglari \\ Department of Computer Engineering, Tehran \\ North Branch, Islamic Azad University, \\ Tehran, Iran
}

\author{
Alireza Bagheri \\ Department of Computer Engineering, Tehran \\ North Branch, Islamic Azad University, \\ Tehran, Iran \\ Department of Computer Engineering and \\ Information Technology, Amirkabir University of \\ Technology, Tehran, Iran
}

\begin{abstract}
Clustering is one of the major techniques in data mining. PreDeCon is a density-based clustering algorithm for computing clusters of spatial objects. In this paper, PPreDeCon is presented as a parallel version of this algorithm in shared memory model. The theoretical analysis and experimental results show that PPreDeCon offers nearly linear speedup while keeps other advantages of PreDeCon.
\end{abstract}

\section{General Terms}

Data mining, parallel algorithms, clustering.

\section{Keywords}

clustering algorithms, parallel algorithms, spatial databases, density-based clustering, shared memory model.

\section{INTRODUCTION}

There is a mixture of data in spatial databases and it is needed to use data mining methods to extract useful information. One of the most important techniques in data mining is clustering. One branch of the clustering methods is density-based clustering branch. Recently, high dimensional and large amount of data in spatial databases are major challenges. Clustering algorithms are categorized into these main types[1]: Partitioning, Hierarchical, Density-based, Gridbased and Model-based algorithms.

In recent years, a number of clustering algorithms have been proposed. One of the most common clustering algorithms is density-based. Main purpose of density-based clustering algorithm is to find and separate high density regions from low density regions. This approach helps algorithm to determine clusters.

There are different algorithms for Density-based clustering approach. DBSCAN is a well-known Density-based algorithm [2]. GDBSCAN extends DBSCAN to determine polygons [3].

OPTICS [4] has been proposed to reduce parameters which are needed for DBSCAN and combined density-based clustering and hierarchical clustering. FDC [5] is a fast density-based clustering algorithm. In this algorithm, the clustering is based on equivalence relationship between objects in database.

Nowadays, due to the fact that a large amount of data must be analyzed, the algorithm should perform effectively in time complexity. In order to improve time complexity of existing algorithms, parallelization is considered. Parallel and distributed computing have an important role in reduction of the response time. With parallel computing, the performance of clustering can be improved. Accordingly, parallel clustering algorithms have been developed and implemented [6]. For Example, PDBSCAN [7] with master-slave configuration has proposed. This algorithm apportions data between processors, each processor clusters data using DBSCAN and in final step, the local clusters merge into global clusters for whole data. Another parallel algorithm is PFDC [8], a parallel algorithm for fast density-based clustering in large spatial databases. It uses MPI and idea of buffering.

High-dimensional data is another problem which needs a solution to tackle it. PreDeCon [9] is a density-based algorithm for computing clusters in moderate-to-high dimensional feature spaces with time complexity of $O\left(d . n^{2}\right)$ ( $d$ is the number of dimensions). In this paper, PPreDeCon algorithm is presented, a parallel version of PreDeCon [9] algorithm with time complexity of $O\left(\frac{d \cdot n^{2}}{p}\right)$. The number of processors is denoted by $P$.

The remaining of the paper is organized as follows. In section 2 the preliminaries are given. In section 3, PreDeCon algorithm is described and section 4 presents the parallel algorithm, PPreDeCon. Section 5 shows the experimental results. Section 6 lists the conclusions and highlights the future works.

\section{PRELIMINARIES}

PreDeCon [9] uses the notions of density-based clusters, mentioned in DBSCAN [2] and this algorithm utilizes subspace clustering concepts [9]. In order to determine which dimensions are relevant to a cluster, the notion of subspace preference for each point is used. In general, a subspace preference cluster is a set of density connected points which are related to a subspace preference vector. Points with small variances are needed to identify subspace preference clusters. Concepts and notions of this section come from [9]. The following definitions are used to clarify PreDeCon. 


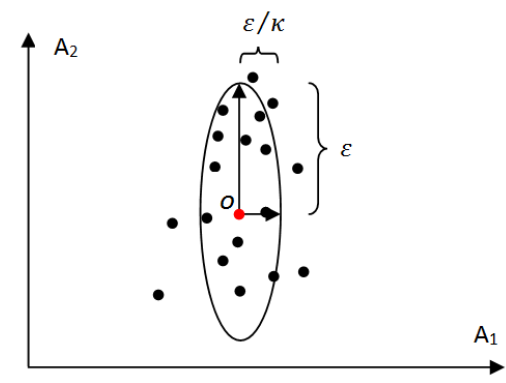

Fig 1: $o$ is a preference weighted core point

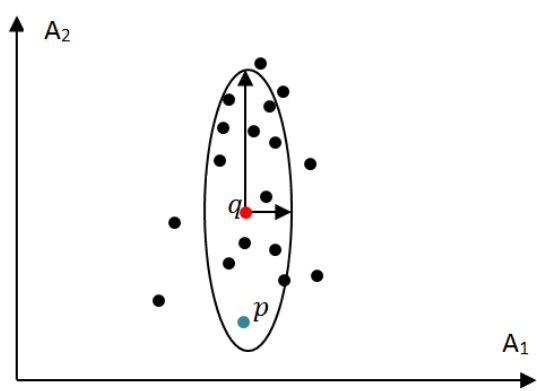

Fig 2: $p$ is directly preference reachable from point $q$

PreDeCon has four input parameters, two density parameters $\varepsilon$ and $\mu$ and two preference parameters $\lambda$ and $\delta$. The parameter $\lambda \in N$ specifies the preference dimension of the subspace preference clusters to be computed. The parameters $\varepsilon \in R$ and $\mu \in N$ specify the density threshold which clusters must exceed [9]. They should be chosen as suggested in [9].

Let $D$ be a database of d-dimensional points $\left(D \subseteq R^{d}\right.$ ), where the set of attributes is denoted by $A=\left\{A_{1}, A_{2}, \ldots, A_{d}\right\}$, and dist $: R^{d} \times R^{d} \rightarrow R$ is a metric distance function between points in $D$.

Let $\mathcal{N}_{\varepsilon}(p)$ be the $\varepsilon$-neighborhood of $p \in D$, i.e., $\mathcal{N}_{\varepsilon}(\mathrm{p})$ contains all points $q \in D$ with $\operatorname{dist}(p, q) \leq \varepsilon$. The variance of $\mathcal{N}_{\varepsilon}(p)$ along an attribute $A_{i} \in A$ is denoted by $V_{A R_{A_{i}}}\left(\mathcal{N}_{\varepsilon}(p)\right)$. Attribute $A_{i}$ is considered a preferable (relevant) dimension for $p$ if the variance with respect to $A_{i}$ in its neighborhood is smaller than a user-defined threshold, i.e. $V_{A R_{A_{i}}} \leq \delta$. All preferable attributes of $p$ are accumulated in the so-called subspace preference vector. This $d$-dimensional vector $\bar{W}_{p}=\left(w_{1}, w_{2}, \ldots w_{d}\right)$ is defined such that $w_{i}=1$ if attribute $\mathrm{A}_{\mathrm{i}}$ is irrelevant, i.e., $V_{A R_{A_{i}}}\left(\mathcal{N}_{\varepsilon}(p)\right)>\delta$ and $w_{i}=\mathcal{K}(\mathcal{K} \gg$ 1) if $A_{i}$ is relevant, i.e. $V_{A R_{A_{i}}}\left(\mathcal{N}_{\varepsilon}(p)\right) \leq \delta$.

The subspace preference vector of points defines the preference weighted similarity function associated with a point $p, \operatorname{dist}_{p}(p, q)=\sqrt{\sum_{i=1}^{d} w_{i} \cdot\left(\pi_{A_{i}}(p)-\pi_{A_{i}}(q)\right)^{2}}$, where $w_{i}$ is the $i$-th component of $\overline{\mathrm{W}}_{\mathrm{p}}$ and $\pi_{A_{i}}(p)$ is the projection of point $p$ onto an attribute $A_{i}$. Using the preference weighted similarity, the preferable attributes are weighted considerably higher than the irrelevant ones. This distance is not symmetric. A symmetric distance is defined by the general preference similarity, $\quad \operatorname{dist}_{\text {pref }}(p, q)=$ $\max \left\{\operatorname{dist}_{p}(p, q), \operatorname{dist}_{q}(q, p)\right\}$.The preference weighted $\varepsilon$ neighborhood of a point $\mathrm{p}$ contains all points of $\mathrm{D}$ that are within a preference weighted distance $\varepsilon$ from $p: \mathcal{N}_{\varepsilon}^{\bar{w}_{o}}(o)=$ $\left\{x \in \mathcal{D} \mid\right.$ dist $\left._{\text {pref }}(o, x) \leq \varepsilon\right\}$. Based on these concepts [9], the classical definitions of density-based clustering have been derived:

Definition 1[9] ( preference dimensionality). Let $p \in D$ and $\delta \in R$. The number of attributes $\mathrm{A}_{\mathrm{i}}$ with $V_{A R_{A_{i}}} \leq \delta$ is called the preference dimensionality of $\mathcal{N}_{\varepsilon}(p)$, denoted by $\operatorname{PDIM}\left(\mathcal{N}_{\varepsilon}(p)\right)$. The intuition of this formalization is to consider those points as core points of a cluster which have enough dimensions with a low variance in their neighborhood.

Therefore, each point $p$ is associated with a subspace preference vector $\bar{W}_{p}$ which reflects the variance of the points in the $\varepsilon$-neighborhood of $p$ along each attribute in $A$.

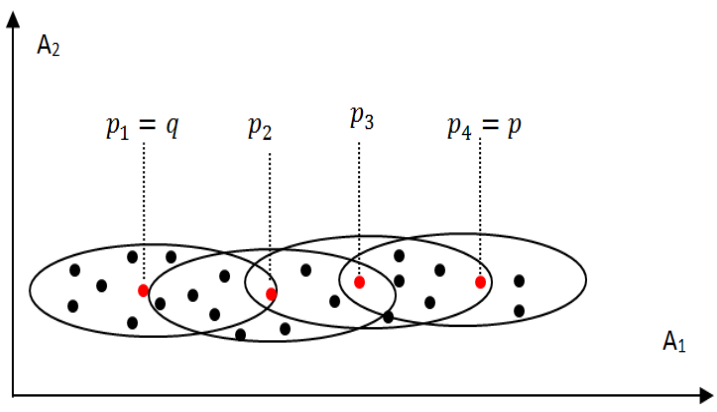

Fig 3: $p$ is preference reachable from point $q$

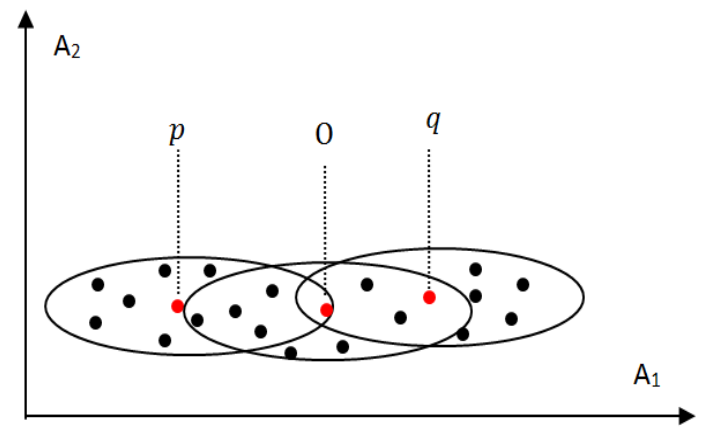

Fig 4: $p$ is preference connected to point $q$

Definition 2[9] (preference weighted core point). A point $o \in D$ is called preference weighted core point w.r.t. $\varepsilon, \mu, \delta$, and $\lambda$ (denoted by $C_{O R E}$ den $(o)$ ), if i) the preference dimensionality of its $\varepsilon$-neighborhood is at most $\lambda$ and ii) its preference weighted $\varepsilon$-neighborhood contains at least $\mu$ points (see fig 1).

Definition 3 [9] ( direct preference reachability). A point $p \in D$ is called directly preference reachable from a point $q \in D$ w.r.t. $\varepsilon, \mu, \delta$, and $\lambda$ (denoted by $D_{I R} R_{E A C H}^{\text {pref }}(q, p)$ ), if $q$ is a preference weighted core point, the subspace preference dimensionality of $\mathcal{N}_{\varepsilon}(p)$ is at most $\lambda$, and $p \in$ $\mathcal{N}_{\varepsilon}^{\bar{w}_{q}}(q)$ (see fig 2).

Definition 4 [9] ( preference reachability). A point $p \in D$ is preference reachable from a point $q \in D$ w.r.t. $\varepsilon, \mu, \delta$, and $\lambda$ (denoted by $R_{E A C H}$ pref $(q, p)$ ), if there is a chain of points $p_{1}, \ldots, p_{n}$ such that $p_{1}=q, p_{n}=p$ and $p_{i+1}$ is directly preference reachable from $p_{i}$ (see fig 3 ).

Definition 5 [9] ( preference connectivity). A point $p \in D$ is preference connected to a point $q \in D$, if there is a point $o \in D$ such that both $\mathrm{p}$ and $\mathrm{q}$ are preference reachable from o (see fig 4). 
Definition 6 [9] ( subspace preference cluster). A non-empty subset $C \in D$ is called a subspace preference cluster w.r.t. $\varepsilon$, $\mu, \delta$, and $\lambda$, if all points in $C$ are preference connected and $C$ is maximal w.r.t. preference reachability.

As DBSCAN, PreDeCon determines a cluster uniquely by any of its preference weighted core points. As far as such a point is detected, the associated cluster is defined as the set of all points that are preference reachable from it [9].

\section{THE PreDeCon ALGORITHM}

To find all subspace preference clusters, the PreDeCon algorithm merely runs one pass over the database according to parameters setting. The pseudo code of the algorithm is given in fig 5. At first, any point of database is marked as unclassified. During running of PreDeCon, noise points are determined and some points get cluster identifications.

The algorithm checks the remained points. If they are preference weighted core points, the algorithm expands the corresponding clusters. Otherwise, those points are determined as noises.

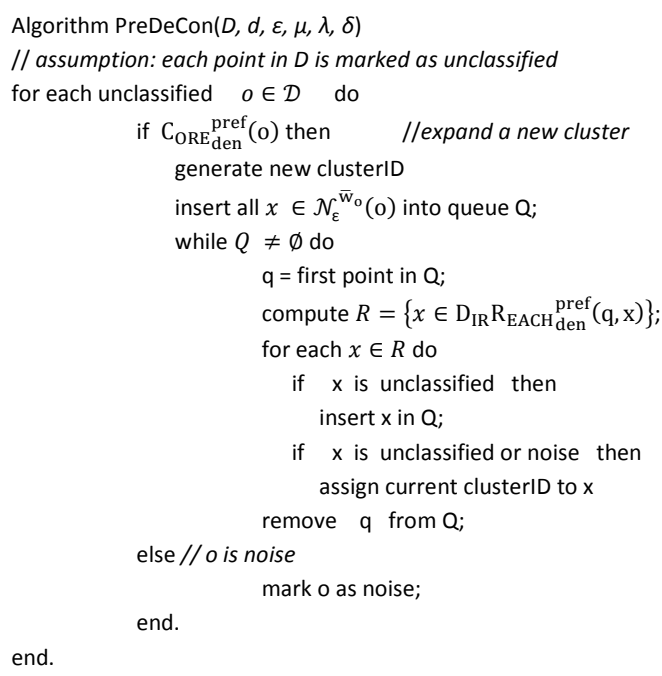

Fig 5: The pseudo code of the PreDeCon algorithm

The algorithm starts with a random preference weighted core point $O$ and generates a new cluster identifier, "clusterID". Then the algorithm looks for all points that are preference weighted reachable from $O$. This clusterID is set for all the points located in the same subspace preference cluster.

The results of PreDeCon algorithm (number of clusters and core points) do not depend on consequent performing. So, the result is deterministic [9].

\section{THE PPreDeCon ALGORITHM}

In our proposed parallel algorithm, all the points are available to each of $P$ processors via the shared memoty. The points are assigned to the processors uniformly in random. The pseudo code of PPreDeCon algorithm is given in fig 6. At first each point is marked as unclassified. Each processor checks its own points. For each point $O$ it is checked whether this point is a preference weighted core point. If so, a new cluster identification (CID) is generated and a new cluster is expanded. Otherwise this point is marked as noise.

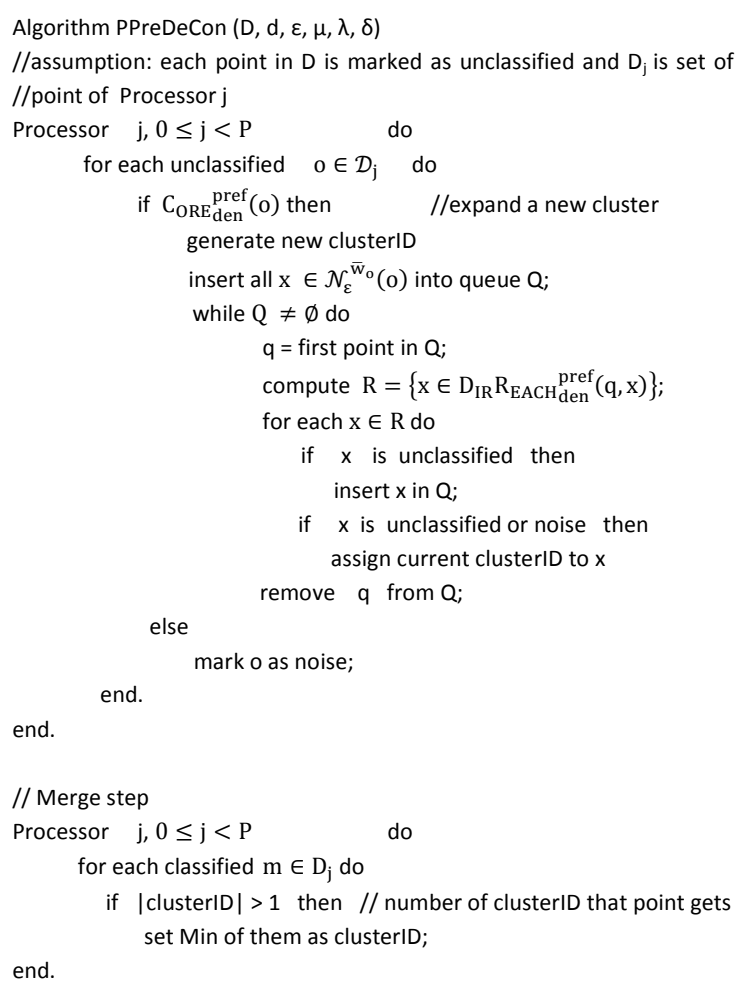

Fig 6: The pseudo code of the PPreDeCon algorithm

The algorithm continues its work with point $O$; and all the points in preference weighted $\varepsilon$-neighborhood of $O$ are inserted into a queue. Then it searches for all directly preference weighted reachable points if they are unclassified, inserts them into the queue. For each point in the queue this is repeated until the queue becomes empty.

After all the processors finish their work, some points may get more than one CID. This problem should be solved. To solve this problem, in merge step, the algorithm sets the minimum CID for each point with more than one CID.

In the following, the time complexity of proposed algorithm is computed. In this parallel algorithm for each point, a preference weighted similarity weight vector is considered. For each point, this vector must be computed once. Computing this vector for each point, considering parallelism can be evaluated in $O\left(\frac{d . n}{P}\right)$ ( $d$ is number of dimensions).

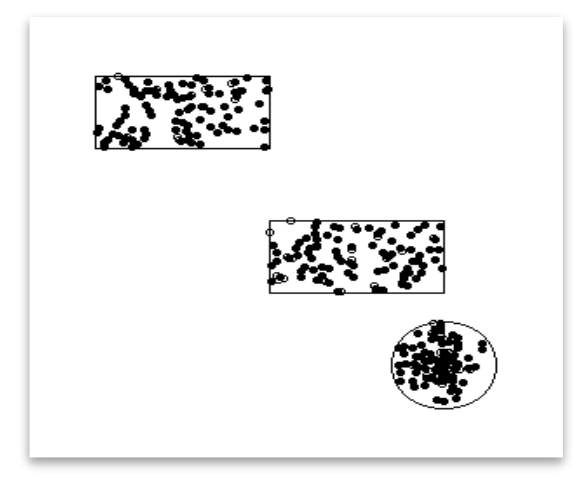

a) Sample database 


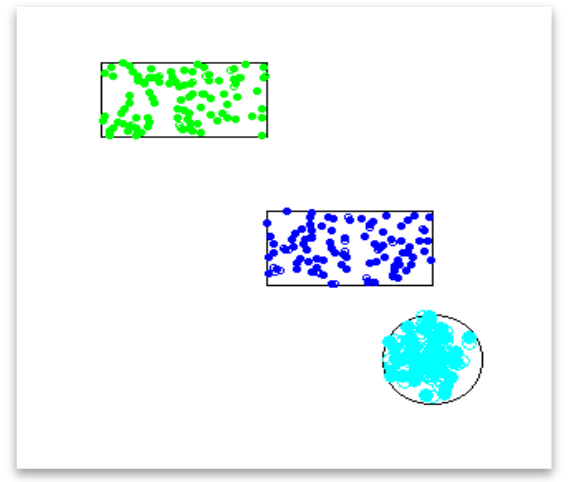

b) Clusters discovered by PPreDeCon

Fig 7: Checking accuracy with sample databases

So computing this vector needs checking the environment of the points in $\varepsilon$-neighborhood along each dimension which is done in $O\left(\frac{d \cdot n}{P}\right)$. Therefore it takes $O\left(\frac{d \cdot n^{2}}{P}\right)$ for all points. According to definition 6 , checking the preference weighted core point and expanding clusters, needs for all point in $\varepsilon^{-}$ neighborhood, evaluation of a weighted distance which can be done in $O\left(\frac{d . n}{P}\right)$. Merge step is done in $O\left(\frac{n^{2}}{P}\right)$ to compute minimum CID for all points. According to above computations, A worst case time complexity of this algorithm is $\left(\frac{d \cdot n^{2}}{P}\right)$. This yields a linear speedup against PreDeCon.

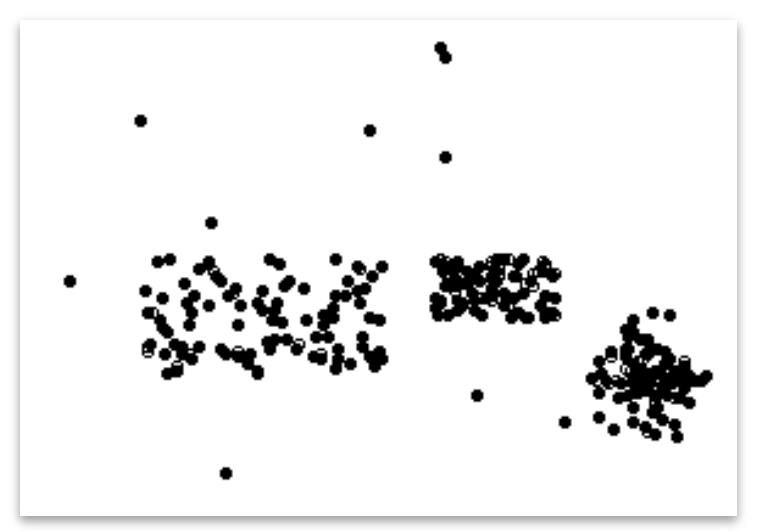

a) Datasets before clustering

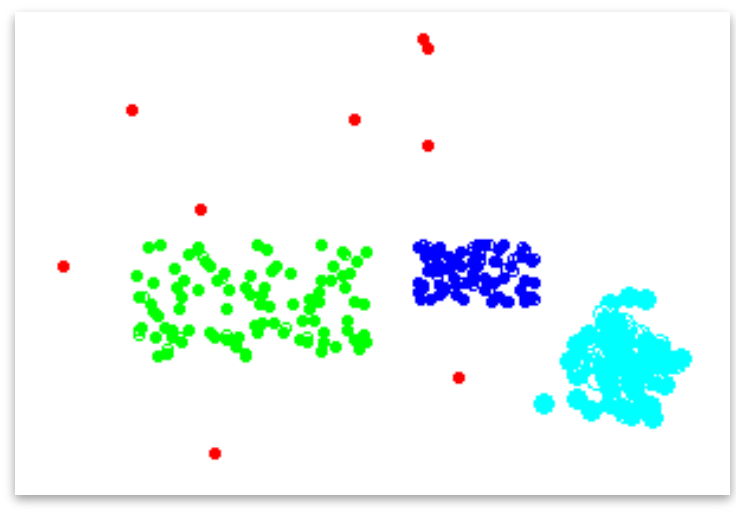

b) Datasets after running proposed algorithm

Fig 8: Running proposed algorithm, PPreDeCon, in Dataset with noise
Table 1. run time of PreDeCon and PPreDeCon

\begin{tabular}{|c|c|c|}
\hline \multirow{2}{*}{$\begin{array}{c}\text { Number of } \\
\text { points }\end{array}$} & \multicolumn{2}{|c|}{ Run time in seconds(P=2) } \\
\cline { 2 - 3 } & PreDeCon & $\begin{array}{c}\text { PPreDeCon } \\
\text { (proposed algorithm) }\end{array}$ \\
\hline D1 =50 & 0.01 & 0.009 \\
\hline D2 =100 & 0.03 & 0.021 \\
\hline D3 = 250 & 0.08 & 0.04 \\
\hline D4 =300 & 0.09 & 0.054 \\
\hline D5 = 500 & 0.21 & 0.12 \\
\hline
\end{tabular}

\section{EXPERIMENTAL EVALUATION}

In this section, accuracy and time-complexity of PPreDeCon are evaluated. For the evaluation, randomly generated data points within three clusters are used. It is depicted in fig 7. The dimension of the data sets is chosen as two. As it is shown in fig 7 , the data points are randomly generated inside two rectangles and a circle.

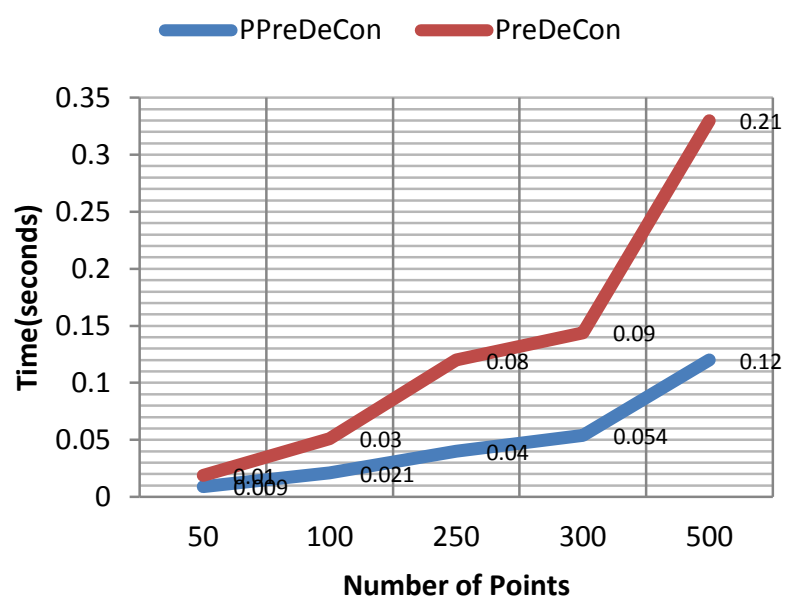

Fig 9: Run time comparison between PreDeCon and PPreDeCon

In all experiments PPreDeCon discovered all the clusters and detected noises as good as PreDeCon. Lots of databases are used in these experiments which one of them is depicted in fig 8 -a. This sample database contains three clusters with different shapes and sizes with additional noise. To show the result, each cluster is visualized by a different color. The result is depicted in fig 8-b. All clusters and noises discovered correctly.

Five 2-dimensional sample datasets are used to evaluate the run time of proposed algorithm. The experimental results are summarized in table 1 . In these experiments the number of processors $P$ is 2 . The observation of the result shows that the run time of PPreDeCon is extremely lower than PreDeCon which is compared in fig 9 .

\section{CONCLUSIONS}

In this paper, a parallel clustering algorithm, PPreDeCon, is presented for mining large and high dimensional spatial databases. Lack of time can be solved by parallel computing. The parallel implementation uses shared memory model. The experiments showed that the actual clustering could be performed with good speed-up and good response time. 
As other advantages of this algorithm are accuracy and supporting high dimensional datasets and compared to other density-based clustering algorithms, it discovers the arbitrary shapes and is effective in noise detecting.

Future research may consider the following issues. Shared memory model is used for parallelism. However one may consider message passing model and also distributed memory. Concentration will be on density-based approaches. The parallelization of other spatial data mining methods may be considered in future. Computing times of all steps of the algorithm and focusing on reducing them maybe appropriate as future works.

\section{REFERENCES}

[1] D. Joshi, A.K. Samal, and Leen-Kiat Soh, "Densitybased clustering of polygons," Computational Intelligence and Data Mining: CIDM'09, IEEE, pp.171178,2009

[2] M. Ester, HP. Kriegel, J. Sander, X. Xu, "A densitybased algorithm for discovering clusters in large spatial databases with noise," In Proc. 2nd Int. Conf. on Knowledge Discovery and Data Mining: KDD, Vol.96, pp.226-231, 1996.

[3] J. Sander, M. Ester, HP Kriegel and X.Xu, "Densitybased clustering in spatial databases: The algorithm GDBSCAN and its Applications," Data Mining and Knowledge Discovery, Vol.2, Iss.2, pp. 169-194, 1998.
[4] M. Ankerst, MM. Breunig, HP. Kriegel and J. Sander, "OPTICS: ordering points to identify the clustering structure," ACM SIGMOD Record, Vol. 28, Iss.2, pp. 4960, 1999.

[5] B. Zhou, D. W. Cheung and B. Kao, "A fast algorithm for density-based clustering in large database," Methodologies for Knowledge Discovery and Data Mining, Springer Berlin Heidelberg, Vol. 1574, pp. 338349, 1999.

[6] Kim, Wooyoung, "Parallel clustering algorithms: survey," CSC 8530 Parallel Algorithms, spring 2009.

[7] X. Xu, J. Jager, and HP. Kriegel, "A Fast Parallel Clustering Algorithm for Large Spatial Databases," High Performance Data Mining, Springer, pp. 263 - 290, 2002.

[8] H. Meyerhenke, "PFDC: A Parallel Algorithm for Fast Density-based Clustering in Large Spatial Databases," In Informatiktage 2002, Konradin Verlagsgruppe, pp. 48 52, 2003.

[9] C. Böhm, K. Railing and HP. Kriegel, "Density connected clustering with local subspace preferences," 4th Int. Conf. on Data Mining: ICDM'04, IEEE, pp. $27-$ 34, 2004. 